\title{
JEAN-CHRISTOPHE LALANNE \\ Caractérisation de la charge des arêtes du graphe support d'un réseau nodal de commutation temporelle asynchrone
}

Revue française d'automatique, d'informatique et de recherche opérationnelle. Recherche opérationnelle, tome 22, n 2 (1988), p. 177-203.

$<$ http://www.numdam.org/item?id=RO_1988_22_2_177_0>

(C) AFCET, 1988, tous droits réservés.

L'accès aux archives de la revue « Revue française d'automatique, d'informatique et de recherche opérationnelle. Recherche opérationnelle » implique l'accord avec les conditions générales d'utilisation (http://www.numdam.org/ legal.php). Toute utilisation commerciale ou impression systématique est constitutive d'une infraction pénale. Toute copie ou impression de ce fichier doit contenir la présente mention de copyright.

\section{Numdam}

Article numérisé dans le cadre du programme

Numérisation de documents anciens mathématiques

http://www.numdam.org/ 


\title{
CARACTÉRISATION DE LA CHARGE DES ARÊTES DU GRAPHE SUPPORT D'UN RÉSEAU NODAL DE COMMUTATION TEMPORELLE ASYNCHRONE ( $\left.{ }^{*}\right)$
}

\author{
par Jean-Christophe LALANNE $\left({ }^{1}\right)$
}

\begin{abstract}
Résumé. - La conception de nouvelles architectures en vue du RNIS (Réseau Numérique à Intégration de Service) peut s'appuyer sur une solution originale de commutation autorisant des applications multidébits et multiservices: la commutation temporelle asynchrone. Sur cette base, des études menées par Alcatel ont développé la notion de structure nodale répétitive, de sorte que le commutateur peut s'identifier à un graphe interconnectant des nouds élémentaires de commutation. On parle alors de "Réseau Nodal Asynchrone " (RNA).

Cet article présente une méthode opérationnelle d'analyse de la charge des liaisons inter-nouds. En partant de la structure topologique du graphe support d'un RNA, on définit puis on calcule des facteurs topologiques dont la simplicité d'usage autorise plusieurs applications : dimensionnement, routage, extension, choix de structure, etc.

Cette modélisation est généralisable à tout graphe dont les nœuds injectent du trafic à destination des autres nouds, ce trafic se répartissant sur toutes les arêtes du graphe.
\end{abstract}

Mots clés : Graphes, plus court chemin, couplage, routage, flots, caractérisation du trafic.

Abstract. - New architectures conception in the ISDN, (Integrated Services Digital Network) find and original switching solution which implements multirates and multiservices applications: asynchronous time division switching. Using this technique Alcatel has studied a nodal structure so that the exchange looks like a graph of which nodes are elementary switching matrices. Such a graph is so called "Asynchronous Nodal Network" (ANN).

This paper presents an operational method to compute inter-nodes linkloads. Topological structure of the ANN support graph allows to define and calculate topological factors of which applications are: sizing, routing, graph extension, structure choice etc.

This method is applicable to every graph of which nodes are injecting traffic towards the other nodes and through the whole network.

Keywords : Graphs, Shortest path, Matching, Routing, Flows, Traffic characterization.

\section{INTRODUCTION}

Les réseaux d'interconnexion de processeurs soht le prolongement logique des rapides progrès, réalisés depuis plusieurs années en matière de technologie informatique.

(*) Reçu juin 1987.

(1) SEMA-METRA, 16-18, rue Barbès, 92126 Montrouge Cedex (Article rédigé dans le cadre d'une Étude Alcatel Commutation Publique). 
Dans la référence [1], un certain nombre d'articles présentent plusieurs implémentations possibles.

Les systèmes multi-processeurs répartissant à la fois la commande et les ressources, ont été naturellement introduits en télécommunications [2, 3]. D'autre part, les techniques utilisant la commutation de paquets pour transmettre des données, ont été appliquées à ces réseaux d'interconnexion $[4,5]$.

Les études menées en commutation temporelle asynchrone résultent de l'imbrication de ces 2 concepts.

Elles ont montré qu'il était possible de réaliser aussi bien des réseaux d'interconnexion de processeurs que des organes de commutation dans la perspective de l'intégration des réseaux de communication $[6,8]$.

\subsection{Technique temporelle asynchrone}

Cette technique de commutation et de multiplexage est présentée dans les références $[6,7]$. On peut brièvement la décrire par les caráctéristiques suivantes.

Le support de transmission, partagé temporellement, ménage des espaces temporels banalisés, de durée fixe ou variable à l'intérieur desquels va s'insérer l'information à transmettre identifiée nécessairement par un en tête, le multiplexage n'étant pas synchrone. On parle alors de paquets (fig. 1). Le terminal ou la station désirant émettre du trafic sur le support vers un destinataire

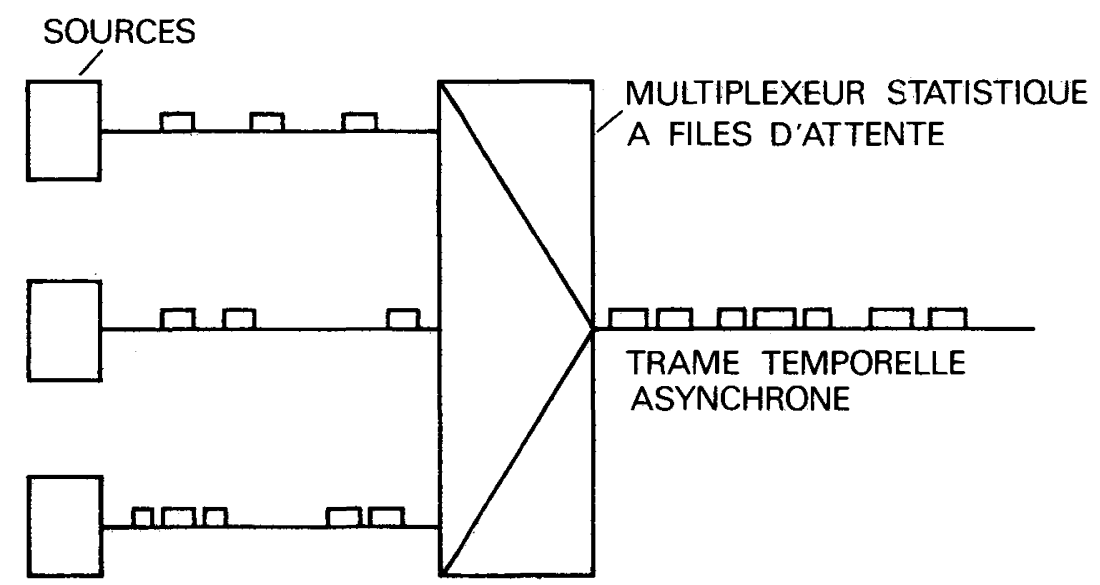

Figure 1. - Multiplexage temporel asynchrone. 
connu, définit le débit réel transmis : ce débit peut être quelconque, variable, inférieur cependant au débit de transmission (en valeur moyenne). Cet indéterminisme d'injection du trafic des sources implique un mécanisme de stockage associé à chaque entrée et sortie du système [8] :

Les paquets désirant emprunter la même sortie sont stockés dans une file d'attente qui résout la contention d'accès aux supports de transmission.

Un réseau de commutation est constitué de $N$ sources, dont le trafic multiplexé en mode temporel synchrone va devoir pour être acheminé, être commuté à l'aide d'un réseau de matrices ayant pour entrée-sortie, des multiplex temporels asynchrones. Le réseau de commutation est donc un ensemble maillé de files d'attente [9].

Le dimensionnement d'un Réseau Temporel Asynchrone passe donc par la résolution des calculs de files d'attente [10]. Ces calculs sont basés sur la connaissance de 2 lois de distribution :

- loi de distribution des longueurs de paquets;

- loi de distribution des arrivées de paquets.

En fait, la connaissance aussi complète que possible des caractéristiques de trafic sur le support, suffit en général pour dimensionner le Réseau de files d'attente et pour calculer les temps de transit.

Or ce trafic se concrétise lui même par l'évolution de la charge du support, définie par le rapport

$$
\frac{\text { débit réel écoulé par le support }}{\text { débit de transmission du support }} \text {. }
$$

L'hypothèse du mode "cut through " [11] permet d'identifier la charge moyenne d'une maille au taux d'occupation de la file d'attente qui lui est affectée [10]. Le taux d'occupation permet de dimensionner la file d'attente.

En mode " cut through " la durée de service d'un paquet dans une file est sa durée de transmission sur le multiplex de sortie.

Dans cet article on propose une méthode de calcul de la charge du support et de ses variations, dans le cas d'un réseau de commutation temporelle asynchrone à architecture nodale.

\subsection{Réseau nodal asynchrone}

Afin de concilier l'architecture distribuée et le concept de commutation temporelle asynchrone, Alcatel a entrepris l'étude d'un réseau expérimental original appelé Réseau Nodal Asynchrone (RNA) [11, 12]. 
Un RNA a pour support un graphe [13] dont chacun des nœuds remplit 3 fonctions. En effet, si l'on considère qu'un équipement d'extrémité peut se comporter comme un ensemble de sources de trafic (c'est-à-dire émetteur de trafic) ou de terminaux de trafic (c'est-à-dire récepteur du trafic qui lui est destiné), il est associé à un nœud du RNA qui :

- écoule le trafic issu des sources qu'il identifie dans le graphe support. Le trafic est paquétisé puis dirigé vers les nœuds destinataires, grâce au réseau de files d'attente;

- reçoit le trafic destiné aux terminaux qu'il identifie dans le graphe support;

- fait transiter le trafic émis (par une source) qui doit l'utiliser en transit pour joindre un destinataire non voisin (adjacent) ( fig. 2).

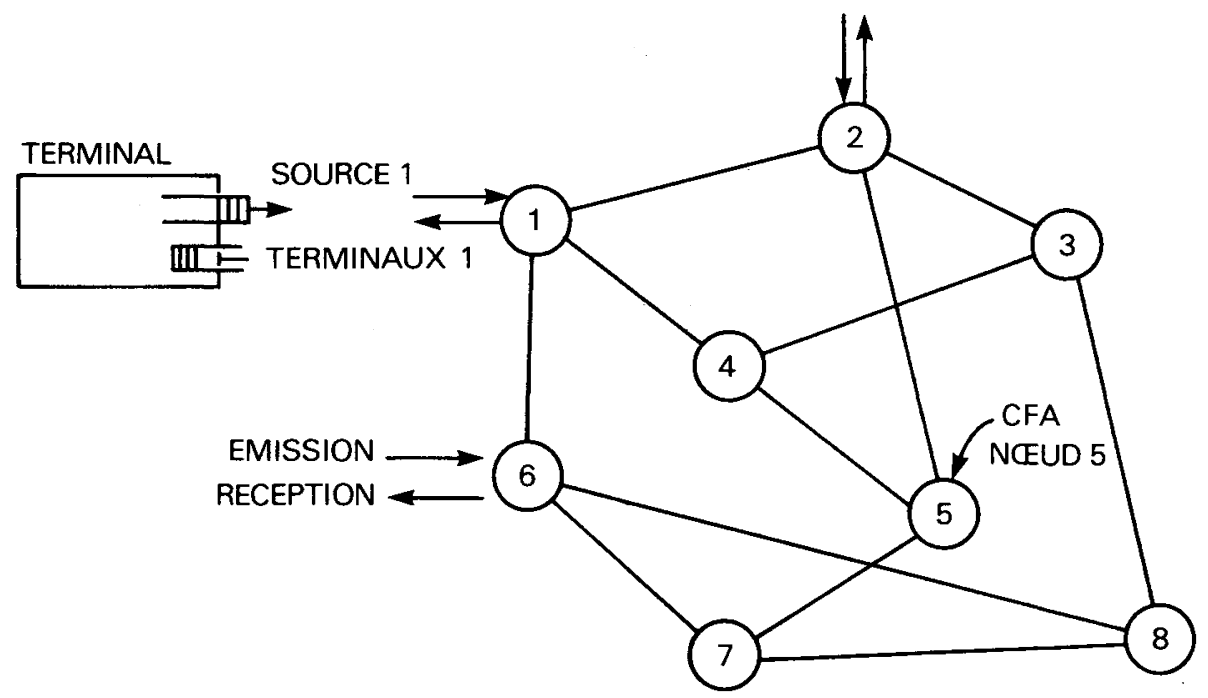

Figure 2. - Réseau nodal.

Chaque nœud de commutation est construit autour d'un commutateur à files d'attente (CFA), permettant de stocker les paquets dirigés vers une même sortie (voir fig. 3).

Les nœuds sont reliés par des supports bidirectionnels appelés maille ou arête.

Ces mailles sont donc parcourues par un trafic dont la charge permettra de dimensionner le CFA, tout en évaluant les capacités du réseau en matière d'efficacité, d'extensibilité et de sécurité. 


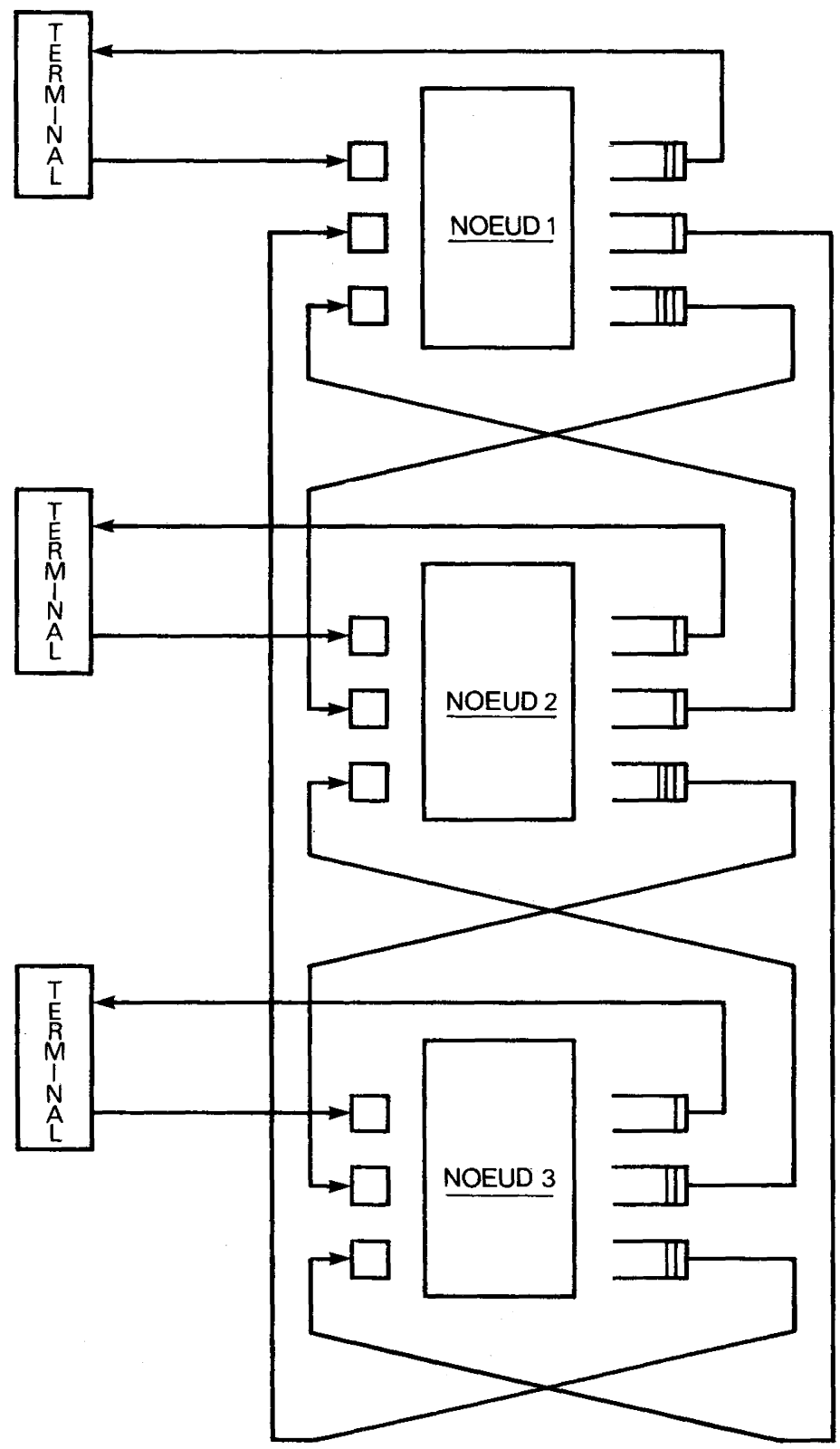

Figure 3. - Réseau de 3 commutateurs à file d'attente. 


\section{Hypothèses}

Un certain nombre d'hypothèses seront appliquées par la suite.

- Chaque nœud injecte dans le réseau et reçoit des autres nœuds un même débit maximal noté Dinj.

- Le routage permettant de relier 2 nœuds entre eux cherche un plus court chemin $(\mathrm{PCCH})$ en nombre d'arêtes utilisées.

\subsection{Réseau nodal asynchrone et théorie des graphes}

On rappelle brièvement les définitions nécessaires à la lecture de cet article. On les illustre par un graphe exemple qui sera utilisé par la suite.

Un noud est un élément du graphe support du RNA.

Une maille (ou arête) est une liaison physique bidirectionnelle entre deux nœuds. L'ensemble des mailles est $\mathscr{M}$.

Un graphe est un ensemble de nœuds, $\mathscr{N}$, reliés par des mailles de $\mathscr{M}$.

$L$ 'ordre $N$ d'un graphe est le nombre de ses nœuds. Le nombre de mailles est noté $M$.

Deux nœuds sont adjacents si une maille les relie.

Le degré $p_{A}$ d'un nœud $A$ est le nombre des nœuds qui lui sont adjacents. Le degré du graphe est le $p_{a}$ maximum, $p$.

La distance $d(A, B)$ entre $A$ et $B$ est le nombre minimal de mailles à emprunter pour relier $A$ à $B$.

Le diamètre $T$ d'un graphe est la borne supérieure des distances entre les nœuds.

La distance moyenne entre deux nœuds $\bar{T}$, fournit le diamètre moyen. Le degré moyen du graphe est la moyenne des degrés; il est noté $\bar{p}$.

La structure d'un graphe est la loi de raccordement des nœuds entre eux. Elle permet de calculer les caractéristiques du graphe.

Les caractéristiques d'un graphe sont données $\operatorname{par}(N, T, \bar{T}, p, \bar{p})$.

Deux graphes ayant mêmes caractéristiques n'ont pas nécessairement la même structure.

$$
\begin{gathered}
\text { Structure } \Rightarrow \text { caractéristiques } \\
\text { (pas d'équivalence) }
\end{gathered}
$$


Exemple :

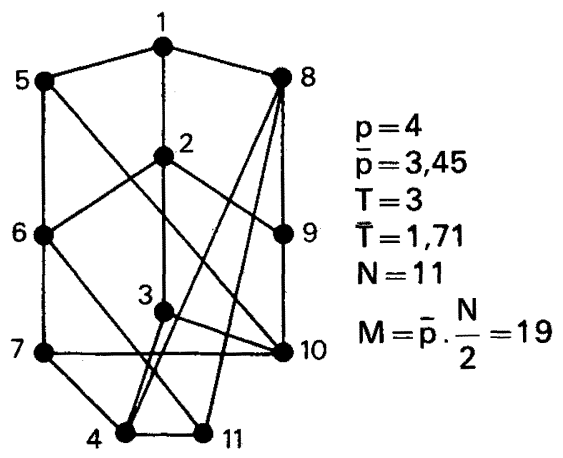

\subsection{Objectifs de l'étude}

Les structures topologiques des graphes ont fait l'objet de nombreuses études [14, 19], le but consistant à optimiser l'un des paramètres du graphe, les autres étant fixés.

Les calculs de flots dans les graphes [15] essayent le plus souvent d'optimiser l'utilisation de la capacité des arêtes. Enfin, les études de routage dynamique ou statique, cherchent à réduire les déséquilibres du trafic.

La démarche opérationnelle proposée consiste à déterminer la charge de chacune des mailles du graphe support en fonction de la structure du graphe, du type de trafic échangé et de la stratégie de routage.

Il sera alors possible de dimensionner chacune des files d'attente situées en amont de chacune des mailles du réseau, et de déterminer le débit de transmission compatible avec les performances attendues.

\section{FACTEURS TOPOLOGIQUES}

\subsection{Principe général}

Le principe général de l'étude consiste à définir un algorithme de calcul de la charge moyenne de chacune des arêtes du graphe, connaissant :

- la distribution générale des trafics échangés

- les plus courts chemins et leur probabilité d'être retenus.

L'idéal serait de disposer d'une part d'un facteur simple relatif à chaque maille et d'autre part d'une borne supérieure de ces facteurs qui, multipliés

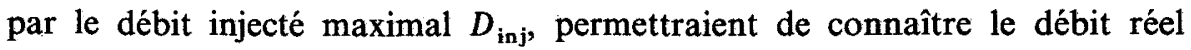
écoulé sur chaque maille et une borne supérieure de ce débit. Si $F$ est ce 
facteur, $Y$ la charge et $\rho$ le rendement dû au mode d'acheminement des paquets (rapport du nombre d'éléments binaires utiles/nombre d'éléments binaires transmis), et si

$D_{\text {trans }}$ est le débit de transmission du support, la formule suivante se déduit de 1.1:

$$
Y=\left(D_{\mathrm{inj}} \cdot \frac{1}{\rho} \cdot F\right) / D_{\mathrm{trans}}
$$

$\left(D_{\text {inj }}\right.$ et $D_{\text {trans }}:$ même unité ex. : kbits/sec.)

\subsection{Définitions}

On appelle facteur topologique $F(A, B)$ relatif à la maille $(A, B)$, le paramètre qui, pour un graphe de structure connue et sous des hypothèses précises de routage et de distribution du trafic échangé entre sources et destinations, donne par simple multiplication du débit injecté sur chaque nœud, le débit réel moyen s'écoulant sur la maille $(A, B)$ :

$$
\text { Débit réel }(A, B)=F(A, B) \times D_{\text {inj }} \times \frac{1}{\rho}
$$

On appelle facteur topologique $F$ du graphe support, le facteur topologique relatif à la maille la plus chargée de ce graphe :

$$
F=\max _{(A, B) \in \mathcal{M}} F(A, B)
$$

Débit réel (maille la plus chargée) $=F \times D_{\mathrm{inj}} \times \frac{1}{\rho}$

Ainsi, le facteur topologique intègre, du point de vue des charges de mailles et des évaluations de performances du RNA, la structure du graphe, le routage utilisé et le trafic échangé.

Il faut noter que cette approche est voisine du calcul présenté dans [16] pour les réseaux delta.

\subsection{Hypothèses possibles}

On recense ici les hypothèses naturelles de trafic et de routage qui permettront d'encadrer le facteur topologique dans un cas quelconque. 


\subsubsection{Trafics}

Ce sont les configurations $T_{m}(m=1,2,3)$ ci-après. On notera $t_{X_{i}, Y_{j}}$ la proportion du Débit injecté sur $X_{i}$ vers $Y_{j}$.

- $i$ et $j$ varient de 1 à $N$ (ordre du graphe).

- $t_{X_{i}, Y_{j}}=0$ si $X_{i}=Y_{j}$.

- Une matrice de trafic symétrique impose $t_{X_{i}, Y_{j}}=t_{Y_{j}, X_{i}}$.

$-\sum_{i=1}^{N} t_{X_{i}, Y_{j}}=1=\sum_{j=1}^{N} t_{X_{i}, Y_{j}}$

T1 : Trafic équiréparti.

Dans ce cas : $t_{X_{i}, Y_{j}}=1 /(N-1)$ pour tout $i$ et tout $j$.

T2: Trafic connu.

L'utilisateur connaît précisément $t_{X_{i}, Y_{j}}$ pour tous les couples de nœuds.

T3 : Trafic pessimiste

Soit un graphe quelconque $(\mathscr{N}, \mathscr{M})$ et une arête $(A, B)$ de ce graphe.

On définit un Couplage maximum relatif à l'arête $(A, B)$ :

Soit $G \subset \mathscr{N}$ l'ensemble des origines et des extrémités des plus courts chemins $(\mathrm{PCCH})$ utilisant $(A, B)$. Construisons le graphe auxiliaire $(G, E)$ bâti autour de $(A, B)$ tel que deux nœuds de $G$ sont reliés par une arête s'il existe entre eux un PCCH utilisant $(A, B): E$ est l'ensemble de ces arêtes.

Un Couplage maximal relatif à l'arête $(A, B)$ est un ensemble $E^{\prime}$ d'ordre maximal des arêtes de $E$ non adjacentes [13] (c'est-à-dire sans arêtes communes).

La distribution de trafic pessimiste sur $(A, B)$ est celle qui maximise le trafic sur $(A, B)$ par échange du trafic maximal entre les origines et les extrémités des arêtes de $E^{\prime}: t_{X_{i}, Y_{i}}=1$ si $\left(X_{i}, Y_{i}\right) \in E^{\prime}$.

Exemple : Soit le graphe suivant

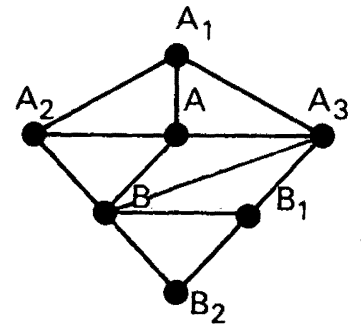

$$
\begin{gathered}
\mathcal{N}=\left\{A_{1}, A_{2}, A_{3}, A, B, B_{1}, B_{2}\right\} \\
G=\left\{A, A_{1}, B, B_{1}, B_{2}\right\}
\end{gathered}
$$

L'étude du couplage maximal relatif à $(A, B)$ donne :

$$
\begin{gathered}
E=\left\{\left(A_{1}, B_{2}\right),\left(A_{1}, B\right),\left(A, B_{1}\right),\left(A, B_{2}\right),(A, B)\right\} \\
E^{\prime}=\left\{\left(A_{1}, B_{2}\right),\left(A, B_{1}\right)\right\}
\end{gathered}
$$


La distribution de trafic pessimiste est celle qui injecte $D_{\mathrm{inj}}$ sur $A_{1}$ vers $B_{2}$ et $D_{\text {inj }}$ sur $A$ vers $B_{1}$.

$$
t_{A_{1}, B_{2}}=1 ; \quad t_{A, B_{1}}=1 .
$$

\subsubsection{Routage}

Ce sont les configurations $R_{n}(n=1,2,3,4)$ :

$R_{1}$ : Routage optimiste.

Ce routage impose l'utilisation de $(A, B)$ entre $X_{i}$ et $Y_{i}$ si tous les PCCH entre $X_{i}$ et $Y_{i}$ passent par $(A, B)$.

\section{$R_{2}:$ Routage équiprobable}

La probabilité d'utiliser $(A, B)$ pour aller de $X_{i}$ à $Y_{i}$ est égale au rapport du nombre $n i$ de PCCH entre $X_{i}$ et $Y_{i}$ utilisant $(A, B)$ au nombre $m i$ de PCCH entre $X_{i}$ et $Y_{i} ; \alpha_{i}=n i / m i$.

$R_{3}$ : Routage connu.

L'utilisateur choisit, selon un algorithme de routage qu'il s'est donné, le chemin utilisé entre $X_{i}$ et $Y_{i}$. Ce chemin n'utilise pas nécessairement $(A, B)$ mais est un $\mathrm{PCCH}$.

$R_{4}$ : Routage pessimiste.

S'il existe au moins un $\mathrm{PCCH}$ entre $X_{i}$ et $Y_{i}$ passant par $(A, B)$, ce routage impose l'utilisation de $(A, B)$ entre $X_{i}$ et $Y_{i}$.

\subsection{Tableau des facteurs topologiques}

Ces facteurs sont définis pour chaque arête $(A, B)$. Leur notation répond à la fonction suivante :

$$
F_{(A, B)}=F_{u}\left(R_{n}, T_{m}\right)
$$

si la maille $(A, B)$ est la maille $u$.

$u$ varie de 1 à $M$ (nombre de mailles).

\begin{tabular}{|c|c|c|c|c|}
\hline \multicolumn{1}{|c|}{$\begin{array}{l}\text { Routage } \\
\text { Trafic }\end{array}$} & $R_{1}$ & $R_{2}$ & $R_{\mathbf{3}}$ & $R_{\mathbf{4}}$ \\
\hline$T_{1} \ldots \ldots \ldots \ldots \ldots \ldots \ldots$ & $C_{0}$ & $B_{0}$ & $D_{0}$ & $A_{0}$ \\
\hline$T_{2} \ldots \ldots \ldots \ldots \ldots \ldots$ & $C_{1}$ & $B_{1}$ & $D_{1}$ & $A_{1}$ \\
\hline$T_{3} \ldots \ldots \ldots \ldots \ldots$ & $C_{2}$ & $B_{2}$ & $D_{2}$ & $A_{2}$ \\
\hline
\end{tabular}

Ainsi : $F_{u}\left(R_{2}, T_{1}\right)=\left(B_{0}\right)_{u}$ 


\subsection{Premières relations}

Dans le cas d'un trafic équiréparti et d'un routage équiprobable le débit réel moyen $\bar{D}$ s'écoulant sur une maille quelconque du réseau est la moyenne des facteurs topologiques $B_{0}$, multipliée par le débit injecté sur chaque nœud $\left(D_{\text {inj }}\right)$ :

$$
\bar{D}=\frac{1}{M} \cdot \sum_{u=1}^{M}\left(B_{0}\right)_{u} \cdot D_{\mathrm{inj}}
$$

où $\left(B_{0}\right)_{u}$ est le facteur $B_{0}$ sur la maille $u$.

Dans ce même cas, la loi de conservation du trafic sur les 2 sens de chaque maille donne l'égalité :

$$
\begin{gathered}
D_{\mathrm{inj}} \cdot \bar{T} \cdot N=2 M \cdot \bar{D} \quad \text { avec } \quad M=\bar{p} \cdot \frac{N}{2} \\
D_{\mathrm{inj}} \cdot \frac{\bar{T}}{\bar{p}}=\bar{D} \\
\frac{1}{M} \cdot \sum_{u=1}^{M}\left(B_{0}\right)_{u}=\frac{\bar{T}}{\bar{p}}
\end{gathered}
$$

$\bar{T}=$ diamètre moyen, $\bar{p}=$ degré moyen.

Par ailleurs, la description des hypothèses permet de déduire pour tout $m \in\{1,2,3\}$ et pour tout $n \in\{1,2,3,4\}$ la série des inégalités suivantes :

$$
\begin{gathered}
C_{0} \leqq F\left(R_{n}, T_{m}\right) \leqq A_{2} \\
F\left(R_{1}, T_{m}\right) \leqq F\left(R_{2}, T_{m}\right) \leqq F\left(R_{4}, T_{m}\right) \\
F\left(R_{n}, T_{m}\right) \leqq F\left(R_{n}, T_{3}\right) \\
F\left(R_{n}, T_{m}\right) \leqq F\left(R_{4}, T_{m}\right) .
\end{gathered}
$$

En effet en gardant une des deux hypothèses constantes $\left(T_{m}\right.$ ou $\left.R_{n}\right)$, le facteur topologique obtenu est toujours majoré par le facteur topologique calculé $T_{3}$ ou $R_{4}$.

\subsection{Remarque}

Le trafic $T_{3}$ et les routages $R_{1}$ et $R_{4}$ sont strictement liés à une maille $(A, B)$. En revanche, pour les 2 cas restant, la formule de 2.5 s'applique

$$
\frac{\bar{T}}{\bar{p}}=\sum_{u=1}^{M} \frac{F_{u}\left(R_{n}, T_{m}\right)}{M}
$$

$n=2,3, m=1$.

vol. $22, \mathrm{n}^{\circ} 2,1988$ 


\section{CALCUL DES FACTEURS TOPOLOGIQUES}

Ce paragraphe présente une méthode générale de calcul de chacun des facteurs topologiques, sur une maille $(A, B)$.

Le nombre de nœud du graphe est noté $N$; le degré est noté $p$, le diamètre est noté $T$.

\subsection{Réduction du graphe en un arbre de plus courts chemins, utilisant la maille} $(A, B)$

La caractérisation d'une maille $(A, B)$ nécessite une réduction du graphe en un arbre dont $(A, B)$ est le tronc et qui permet de répertorier l'ensemble $\mathscr{E}(A, B)$ des couples de nœuds joignables par au moins un plus court chemin $(\mathrm{PCCH})$ utilisant $(A, B)$.

\subsubsection{Construction de couches}

Si l'on note $\mathscr{D}_{A}(i)$ l'ensemble des nœuds à distance $i$ de $A\left(\mathscr{D}_{A}(0)=\{A\}\right)$, on définit :

$$
\begin{array}{ll}
\mathscr{D}_{A}^{*}(i)=\mathscr{D}_{A}(i)-\mathscr{D}_{B}^{*}(i-1), & i \leqq T-1 \\
\mathscr{D}_{B}^{*}(i)=\mathscr{D}_{B}(i)-\mathscr{D}_{A}^{*}(i-1), & i \leqq T-1 .
\end{array}
$$

On appelle "couches $A$ » («couches $B »)$ l'ensemble des origines (extrémités) des PCCH utilisant $(A, B)$.

Deux résultats sont facilement démontrables (annexe 1) :

Résultat 1. - Tout couple $(X, Y)$ de nœuds admettant un PCCH qui utilise $(A, B)$ est tel que $X$ appartient à un ensemble $\mathscr{D}_{A}^{*}(i)$ et $Y$ appartient à un ensemble $\mathscr{D}_{B}^{*}(j):$ "couches $A » \subset \mathscr{D}_{A}^{*}$ et " couches $B » \subset \mathscr{D}_{B}^{*}$

Résultat 2. - Les « couches $A$ » et les " couches $B$ » doivent être disjointes deux à deux, c'est-à-dire qu'un nœud appartenant à deux ensembles distincts $\mathscr{D}_{A}^{*}(i)$ et $\mathscr{D}_{B}^{*}(j)$ ne peut être origine ou extrémité d'un PCCH utilisant $(A, B)$.

Le résultat permet de redéfinir les "couches $A$ " et les " couches $B$ ». Si $\mathscr{N}$ est l'ensemble des nœuds du graphe et $\mathscr{E}_{i}$ l'ensemble des nœuds non répertoriés à la profondeur $i$ :

$$
\mathscr{E}_{1}=\mathcal{N}-\{A, B\} ; \quad \mathscr{E}_{i}=\mathscr{E}_{i-1}-\left[\mathscr{D}_{A}^{*}(i-1) \cup \mathscr{D}_{B}^{*}(i-1)\right], \quad i \geqq 2 .
$$

Les couches sont alors :

« couches $A$ »: origines

Pour $i=1$ à $T-1$,

$$
\mathscr{D}_{r}^{*} A(i)=\left[\mathscr{D}_{A}^{*}(i)-\mathscr{D}_{B}^{*}(i)\right] \cap \mathscr{E}_{i} ; \quad \mathscr{D}_{r}^{*} A(0)=\{A\}
$$


« couches $B »$ : extrémités

Pour $j=1$ à $T-1$,

$$
\mathscr{D}_{r}^{*} B(j)=\left[\mathscr{D}_{B}^{*}(j)-\mathscr{D}_{A}^{*}(j)\right] \cap \mathscr{E}_{i} ; \quad \mathscr{D}_{r}^{*} B(0)=\{B\} .
$$

3.1.2. Construction de l'arbre
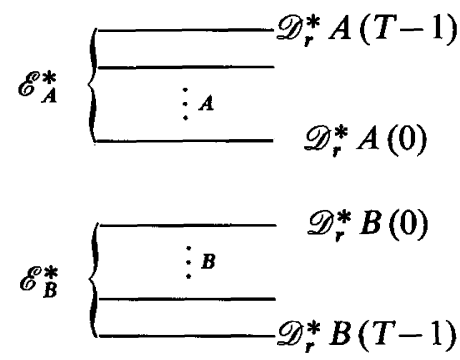

L'ensemble $\mathscr{E}_{A}^{*}$ est l'ensemble des origines et l'ensemble $\mathscr{E}_{\mathscr{B}}^{*}$ celui des extrémités. Le résultat 2 indique :

$$
\mathscr{E}_{A}^{*} \cap \mathscr{E}_{B}^{*}=\varnothing
$$

En conséquence, le graphe tel que $X$ élément de $\mathscr{E}_{A}^{*}$ et $Y$ élément de $\mathscr{E}^{*} B$ sont adjacents si $(X, Y)$ admet un PCCH utilisant $(A, B)$, est un graphe biparti [13]. Il existe des couplages maximaux de $\mathscr{E}_{A}^{*}$ vers $\mathscr{E}_{B}^{*}$ sur ce graphe biparti.

\subsection{Caractérisation d'une maille}

Une maille $(A, B)$ est avant tout caractérisée par l'ensemble $\mathscr{E}(A, B)$ des couples $\left(X_{i}, Y_{i}\right)$ admettant au moins un PCCH utilisant $(A, B)$. Pour déterminer $\mathscr{E}(A, B)$ on utilise l'algorithme :

Pour $j=T-1$ à $j=0\left(j\right.$ distance de $X_{i}$ a $\left.A\right)$.

Pour $X_{i} \in \mathscr{D}_{r}^{*} A(j)$.

Soit $k$ la longueur d'un PCCH d'origine $X_{i}$ et utilisant $(A, B)$.

L'extrémité $Y_{i}$ obtenue par:

$$
\begin{gathered}
k=T \text { à } 1, \\
Y_{i} \in \mathscr{D}_{r}^{*} B(|k-j|) \cap \mathscr{D}_{X_{i}}(k) \text { est telle que }\left(X_{i}, Y_{i}\right) \in \mathscr{E}(A, B) .
\end{gathered}
$$

$n_{i}$ est le nombre de PCCH utilisant $(A, B)$ entre $X_{i}$ et $Y_{i}$.

$m_{i}$ est le nombre total de PCCH entre $X_{i}$ et $Y_{i}$.

$Q_{\text {tot }}=$ Card $\mathscr{E}(A, B) \quad$ [nombre d'éléments de $\mathscr{E}(A, B)$ ].

$Q$ est le cardinal d'un couplage maximal de $\mathscr{E}_{A}^{*}$ vers $\mathscr{E}_{B}^{*}$.

\subsubsection{Exemple}

On étudie la caractérisation de la maille (5.10) du graphe proposé dans le paragraphe 1.3. 


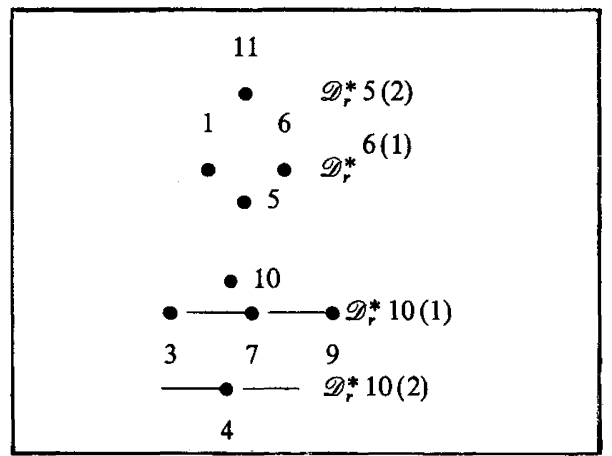

la maille étudiée est :

origine : $5 \rightarrow$ extrémité : 10

couche restreinte entrée d'ordre : 1

16

couche restreinte sortie d'ordre : 1

$\begin{array}{lll}3 & 7 & 9\end{array}$

couche restreinte entrée d'ordre : 2

11

couche restreinte sortie d'ordre : 2

4

le nombre maximal de couples ayant un PCCH empruntant la maille étudiée est : $9=Q_{\text {tot }}$

\begin{tabular}{|c|c|c|c|c|c|}
\hline Origine & Extrémité & Taille & Dist $A$ & Dist $B$ & \\
\hline orig. : 5 & extr. : 10 & tail. 1 & $\operatorname{diA} 0$ & diB 0 & ni $1 \mathrm{mi} 1$ \\
\hline orig. : 5 & extr.: 3 & tail. 2 & $\operatorname{diA} 0$ & $\operatorname{diB} 1$ & ni $1 \mathrm{mi} 1$ \\
\hline orig. : 5 & extr.: 7 & tail. 2 & $\operatorname{diA} 0$ & $\operatorname{diB} 1$ & ni $1 \mathrm{mi} 2$ \\
\hline orig. : 5 & extr. : 9 & tail. 2 & $\operatorname{diA} 0$ & $\operatorname{diB} 1$ & ni $1 \mathrm{mi} 1$ \\
\hline orig. : 5 & extr.: 4 & tail. 3 & $\operatorname{diA} 0$ & $\operatorname{diB} 2$ & ni $2 \mathrm{mi} 5$ \\
\hline orig. : 11 & extr. : 10 & tail. 3 & $\operatorname{diA} 2$ & $\mathrm{diB} 0$ & ni $1 \mathrm{mi} 5$ \\
\hline orig. : 1 & extr. : 10 & tail. 2 & $\operatorname{diA} 1$ & $\operatorname{diB} 0$ & ni $1 \mathrm{mi} 1$ \\
\hline orig. : 6 & extr. : 10 & tail. 2 & $\operatorname{diA} 1$ & $\mathrm{diB} 0$ & ni $1 \mathrm{mi} 2$ \\
\hline orig. : 1 & extr.: 7 & tail. 3 & $\operatorname{diA} 1$ & $\operatorname{diB} 1$ & ni $1 \mathrm{mi} 4$ \\
\hline
\end{tabular}

Le calcul réalisé en PASCAL sur IBM 3083 donne les résultats ci-dessus. 
Le programme permet d'analyser des graphes d'ordre 256 .

Ici :

$$
\begin{gathered}
\mathscr{E}(5,10)=\{(5,9) ;(5,10) ;(5,3) ;(5,4) ;(5,7) ; \\
(1,10) ;(6,10) ;(11,10) ;(1,7)\}
\end{gathered}
$$

$Q_{\text {tot }}=9$. Un couplage possible est $(5,3) ;(6,10) ;(1,7)$. Ce couplage est maximal : $A 2=3$

3.2.2. Borne $Q M d u$ nombre de couples réalisant un couplage maximal

L'application de la méthode précédente aux graphes les plus denses (graphe de Moore) de degré $p$ et diamètre $T(17)$ permet de prouver facilement que

$$
A 2 \leqq Q_{M}=\sum_{i=0}^{T-1} \inf \left((p-1)^{i},(p-1)^{T-1-i}\right) .
$$

Dans l'exemple précédent, $p=4$ et $T=3 \Rightarrow Q_{M}=5$.

\subsection{Formules de calcul des facteurs topologiques d'une maille}

On se reportera aux hypothèses $T_{m}$ et $R_{n}$ formulées dans les paragraphes 2.3 .1 et 2.3.2.

Sous l'hypothèse $R_{n}$, la probabilité pour $\left(X_{i}, Y_{i}\right)$ d'utiliser $(A, B)$, la maille étudiée, sera notée $\left(P_{i}\right)_{R_{n}}$.

Sous l'hypothèse $T_{m}$, la proportion du débit injecté $D_{\text {inj }}$ de $X_{i}$ vers $Y_{i}$ est notée $t\left(X_{i}, Y_{i}\right)_{T_{m}}$.

Par définition du facteur topologique, on obtient la formule fondamentale donnant le facteur topologique $F_{(A, B)}\left(R_{n}, T_{m}\right)$ sous les hypothèses $R_{n}, T_{m}$ pour la maille $(A, B)$ :

$$
\begin{gathered}
F_{(A, B)}\left(R_{n}, T_{m}\right)=\sum_{i=1}^{Q_{\text {tot }}}\left(P_{i}\right)_{R_{n}} \cdot t\left(X_{i}, Y_{i}\right)_{r_{m}} \text { et }\left(X_{i}, Y_{i}\right) \in \mathscr{E}(A, B) \\
\bullet\left(P_{i}\right)_{R_{1}}=\left[\frac{n_{i}}{m_{i}}\right] \quad(\text { partie entière }) \\
\bullet\left(P_{i}\right)_{R_{2}}=\frac{n_{i}}{m_{i}}, \\
\bullet\left(P_{i}\right)_{R_{3}}=\left\{\begin{array}{cc}
1 & \text { si le routage entre } X_{i} \text { et } Y_{i} \text { impose }(A, B) \\
0 & \text { sinon }
\end{array}\right.
\end{gathered}
$$




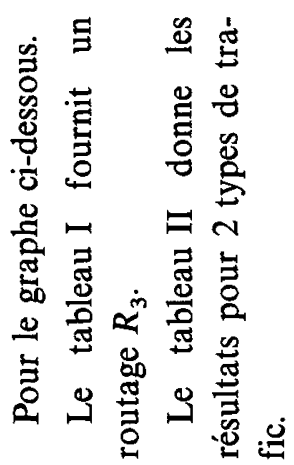

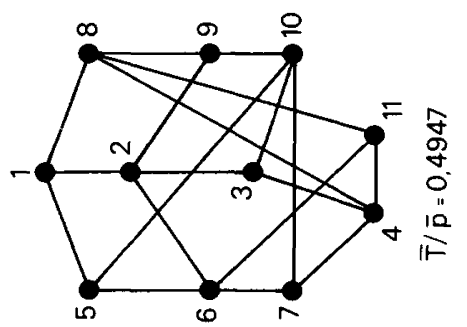

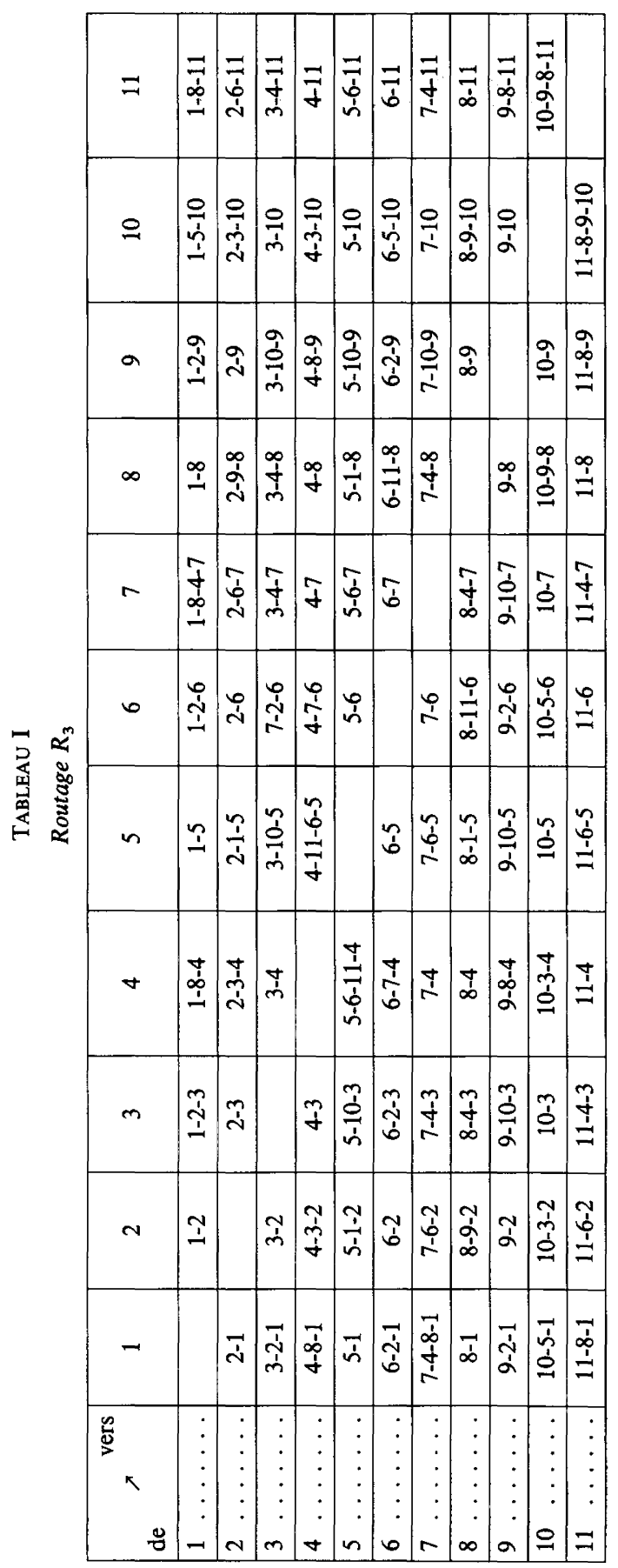

Recherche opérationnelle/Operations Research 


$$
\begin{gathered}
\bullet\left(P_{i}\right)_{R_{4}}=1 \\
\qquad t\left(X_{i}, Y_{i}\right)_{T_{1}}=\frac{1}{N-1}, \quad t\left(X_{i}, Y_{i}\right)_{T_{2}}=t_{X_{i}, Y_{i}}
\end{gathered}
$$

- $t\left(X_{i}, Y_{i}\right)_{T_{3}}=\left\{\begin{array}{cc}1 & \text { si }\left(X_{i}, Y_{i}\right) \text { élément d'un couplage maximal. } \\ 0 & \text { sinon }\end{array}\right.$

Le couplage maximal retenu doit maximiser $F\left(R_{n}, T_{3}\right)$.

On obtient alors les résultats suivants, pour une maille $(A, B)$, connaissant $\mathscr{E}(A, B)$ :

$$
\begin{aligned}
& \left(A_{0}\right)_{(A, B)}=\frac{Q_{\text {tot }}}{N-1} ; \quad\left(B_{0}\right)_{(A, B)}=\sum_{i=1}^{Q_{\text {tot }}} \frac{n_{i}}{m_{i}} \cdot \frac{1}{N-1} \\
& \left(C_{0}\right)_{(A, B)}=\sum_{i=1}^{Q_{\text {tot }}}\left[\frac{n_{i}}{m_{i}}\right] \cdot \frac{1}{N-1} ; \quad\left(A_{2}\right)_{(A, B)}=A_{2}
\end{aligned}
$$

\subsection{Exemple}

\section{TABLEAU II}

\begin{tabular}{|c|c|c|c|c|c|c|c|c|c|}
\hline & & $R_{4}$ & $R_{3}$ & $R_{2}$ & $R_{1}$ & $R_{4}$ & $R_{3}$ & $R_{2}$ & $R_{1}$ \\
\hline $\begin{array}{l}\text { Maille } \\
\text { étudiée }\end{array}$ & $Q_{\text {tot }}$ & $A_{0}$ & $D_{0}$ & $B_{0}$ & $C_{0}$ & $A_{2}$ & $D_{2}$ & $B_{2}$ & $C_{2}$ \\
\hline$(1,2) \ldots \ldots \ldots$ & 7 & 0,7 & 0,5 & 0,425 & 0,2 & 2 & 2 & 1,5 & 1 \\
\hline$\ldots \ldots \ldots$ & 7 & 0,7 & 0,4 & 0,47 & 0,3 & 2 & 2 & 2 & 2 \\
\hline$\ldots \ldots \ldots$ & 8 & 0,8 & 0,5 & 0,545 & 0,4 & 3 & 2 & 2 & 2 \\
\hline$(2,3)$ & 6 & 0,6 & 0,5 & 0,5 & 0,4 & 2 & 2 & 2 & 2 \\
\hline$\ldots \ldots$ & 8 & 0,8 & 0,6 & 0,625 & 0,5 & 3 & 2 & 2,25 & 2 \\
\hline$(2,9)$ & 6 & 0,6 & 0,4 & 0,4 & 0,2 & 2 & 2 & 1,5 & 1 \\
\hline$\ldots \ldots$ & 8 & 0,8 & 0,6 & 0,54 & 0,4 & 3 & 2 & 2,2 & 2 \\
\hline$\ldots \ldots$ & 8 & 0,8 & 0,5 & 0,44 & 0,2 & 3 & 2 & 1,5 & 1 \\
\hline$\ldots \ldots$ & 9 & 0,9 & 0,6 & 0,485 & 0,2 & 3 & 2 & 1,7 & 1 \\
\hline$(4,8)$. & 7 & 0,7 & 0,6 & 0,545 & 0,5 & 3 & 3 & 2,25 & 2 \\
\hline$(4,11)$ & 6 & 0,6 & 0,4 & 0,36 & 0,2 & 2 & 2 & 1,5 & 1 \\
\hline$(5,6)$. & 9 & 0,9 & 0,5 & 0,485 & 0,2 & 3 & 2 & 1,5 & 1 \\
\hline$(5,10)$ & 9 & 0,9 & 0,5 & 0,585 & 0,4 & 3 & 2 & 2 & 2 \\
\hline$(6,7)$. & 9 & 0,9 & 0,4 & 0,49 & 0,2 & 3 & 2 & 1,7 & 1 \\
\hline$(6,11)$ & 8 & 0,8 & 0,5 & 0,56 & 0,4 & 3 & 3 & 2,2 & 2 \\
\hline$\ldots \ldots$ & 9 & 0,9 & 0,2 & 0,485 & 0,2 & 3 & 1 & 1,7 & 1 \\
\hline$(8,9)$. & 7 & 0,7 & 0,6 & 0,52 & 0,4 & 3 & 3 & 2 & 2 \\
\hline$(8,11) \ldots$ & 5 & 0,5 & 0,5 & 0,42 & 0,4 & 2 & 2 & 2 & 2 \\
\hline$(9,10) \ldots \ldots \ldots$ & 9 & 0,9 & 0,6 & 0,52 & 0,4 & 2 & 2 & 2 & 2 \\
\hline Maximum & 9 & 0,9 & 0,6 & 0,625 & 0,5 & 3 & 3 & 2,25 & 2 \\
\hline Moyenne & 7,63 & 0,763 & $\underline{0,4947}$ & $\underline{0,4947}$ & 0,32 & 2,63 & 2,1 & 1,87 & 1,58 \\
\hline
\end{tabular}

Facteurs topologiques

Trafic équiréparti $T_{1}$

Trafic pessimiste $T_{3}$

vol. $22, \mathrm{n}^{\circ} 2,1988$ 
On constate bien que la moyenne des $D_{0}$ et des $B_{0}$ est égale à $\bar{T} / \bar{P}$. On vérifie également que :

$$
C_{0} \leqq D_{0}<A_{0} \quad \text { et } \quad C_{0} \leqq B_{0}<A_{0} .
$$

Supposons que le trafic soit équiréparti et le routage fixé au départ $R_{3}$. On cherche quel doit être le débit de transmission $D_{\text {trans }}$ à fournir (Tableau I) sachant que :

- le rendement est de 0,85 ;

- la charge maximale tolérable est 0,7 .

Le facteur topologique du graphe est dans ce cas 0,6 (tableau II : $D_{0}$ ).

La formule donnée en 2.1 conduit à :

$$
D_{\text {trans }} \geqq D_{\text {inj }} 1,08
$$

Si le trafic est pessimiste, sous les mêmes conditions nous aurons un facteur topologique de 3 (tableau II $/ D_{2}$ ).

$$
D_{\text {trans }} \geqq D_{\text {inj }} 5,36
$$

\section{APPLICATIONS POSSIBLES}

Les applications sont très nombreuses. On en présente seulement quelques unes, significatives de l'étude des performances d'un RNA.

\subsection{Files d'attentes. Charge des mailles}

Comme il a été exposé au paragraphe 1, le Réseau Nodal Asynchrone (RNA) est un réseau de files d'attente ayant pour support un graphe. Ces files d'attente peuvent être dimensionnées et évaluées grâce à la connaissance de la charge des mailles. Ainsi les résultats attendus sont les suivants :

- Probabilité (Perte d'un paquet dans le réseau) $\simeq(T+1) \times$ probabilité (Perte paquet dans une file).

$$
P \simeq(T+1) . P_{\text {File }}(Y)
$$

$T=$ diamètre du graphe, $Y=$ charge de la maille sortie de la file d'attente.

- Temps de traversée du réseau $=(T+1) \times$ Temps de transit dans une file

$$
W \simeq(T+1) W_{\text {file }}(Y)
$$


Ces calculs sont faits pour la maille la plus chargée du graphe. On peut donc faire intervenir le facteur topologique du graphe, puisque d'après le paragraphe 2.1

$$
Y=\frac{D_{\mathrm{inj}}}{D_{\mathrm{trans}}} \cdot \frac{1}{\rho} \cdot F\left(R_{n}, T_{m}\right)
$$

Exemple: Supposons qu'il s'agisse d'une file du type $M / M / 1$. On trouve dans [10] les formules suivantes :

$$
P \simeq(T+1) Y^{k} \quad W=(T+1) \frac{1}{1-Y}
$$

(Unité : Durée d'écoulement d'un paquet à la vitesse $D_{\text {trans }}$ ); $k=$ taille, en nombre de paquets, d'une file d'attente.

Les courbes de la figure 4 donnent $P$ en fonction de $F\left(R_{n}, T_{m}\right), k$ et $D_{\text {inj }} / D_{\text {trans }}$ étant variables : le couple $\left(k, D_{\text {trans }}\right)$ dimensionne les files d'attente du RNA.

Dans le cas représenté : $T=3$ et $\rho=0,8$.

Les courbes de la figure 5 permettent de dimensionner le réseau de files d'attente, en fournissant $k$ et $D_{\text {inj }} / D_{\text {trans }}, P$ étant fixé et $F\left(R_{n}, T_{m}\right)$ défini par l'analyse topologique du graphe, les hypothèses de routage et le trafic.

Pour le graphe présenté en 1.3:

- si le routage est équiprobable et le trafic pessimiste : $F=B_{2}=2.25$

\begin{tabular}{|l|c|c|c|c|}
\hline & $k=8$ & $k=16$ & $k=32$ & $k=64$ \\
\hline Perte $10^{-3} \ldots \ldots \ldots \ldots \ldots \ldots$ & .125 & .22 & .27 & .32 \\
\hline Perte $10^{-6} \ldots \ldots \ldots \ldots \ldots \ldots$ & .05 & .13 & .22 & .28 \\
\hline Perte $10^{-9} \ldots \ldots \ldots \ldots \ldots \ldots$ & .025 & .09 & .17 & .25 \\
\hline
\end{tabular}

\begin{tabular}{|c|c|c|c|c|}
\hline 7 & $k=8$ & $k=16$ & $k=32$ & $k=64$ \\
\hline Perte $10^{-3}$ & .325 & $\begin{array}{ll}.54 \\
\end{array}$ & .69 & .78 \\
\hline Perte $10^{-6}$ & .13 & .35 & .56 & .7 \\
\hline Perte $10^{-9}$ & .06 & .22 & .45 & .63 \\
\hline
\end{tabular}

- si le routage est pessimiste et le trafic équiréparti : $\mathrm{F}=A_{0}=0.9$

On constate l'influence du facteur topologique et donc du trafic et du routage qu'il caractérise. 


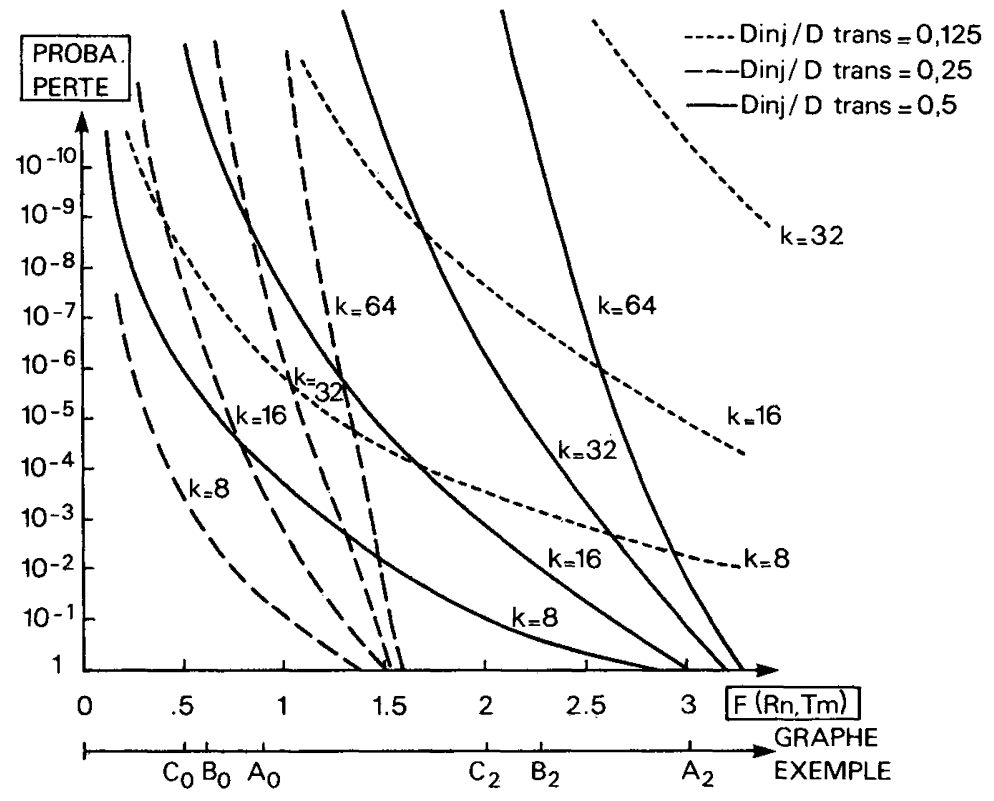

Figure 4

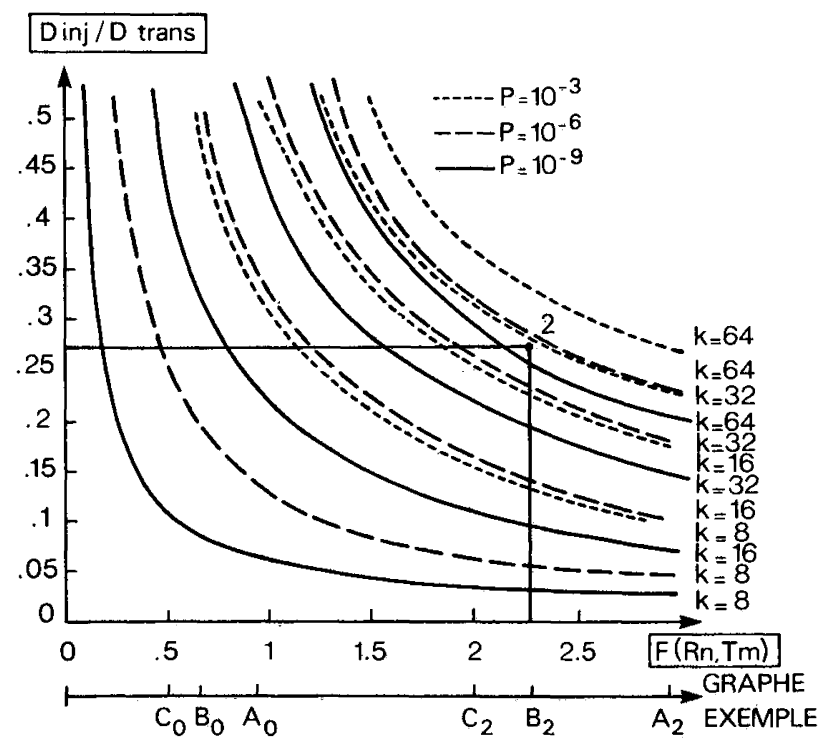

Figure 5 
Des courbes semblables sont obtenues pour le temps de transit ( fig. 6).

Ainsi, si l'on veut obtenir une perte $P \leqq 10^{-6}$ et un temps moyen de transit de moins de 10 temps paquet, dans le cas d'un routage équiprobable, un bon dimensionnement du système donne :

$$
D_{\text {inj }} / D_{\text {trans }} \leqq .25 \text { ( fig. 6, Point } 1 \text { ) et } k \geqq 43 \text { (fig. 5, Point 2). }
$$

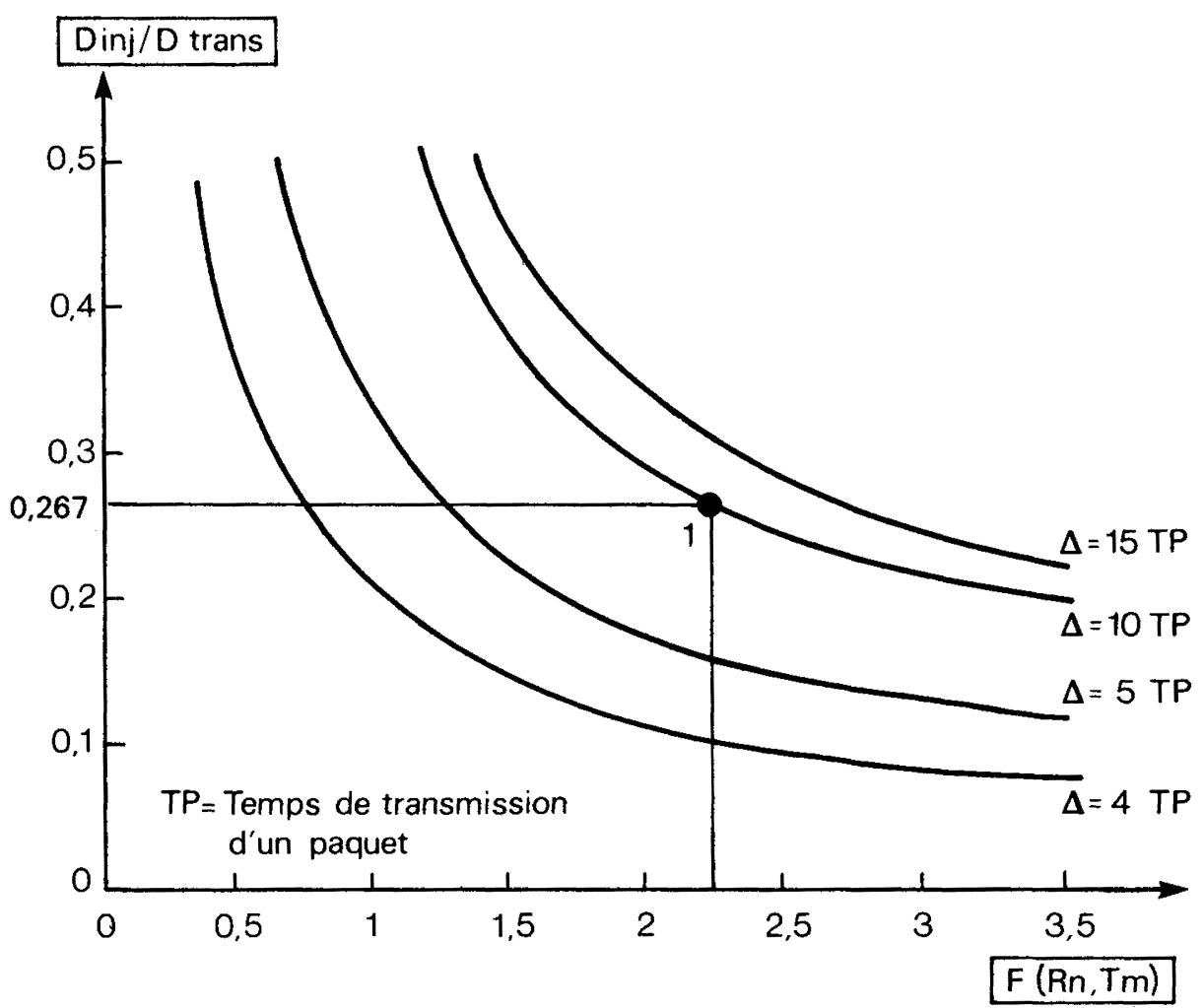

Figure 6. - Caractéristique du temps moyen de transit d'un paquet à travers le graphe 1.3 (diamètre $T=3$ ).

\subsection{Appréciation du trafic et du routage}

\section{Variation de trafic}

La connaissance des variations de trafic et de distribution de trafic, se traduit directement par une variation du facteur topologique et donc par une loi de distribution de la charge moyenne de la maille la plus chargée. 
Qualification d'algorithme de routage

Grâce au facteur topologique on peut efficacement qualifier les capacités d'un algorithme de routage en comparant :

les facteurs topologiques obtenus par plusieurs algorithmes ou le facteur topologique fourni par un algorithme par rapport aux hypothèses $R_{1}, R_{2}$ ou $R_{4}$.

\subsection{Choix d'un graphe extension}

Les critères de choix d'un graphe reposent le plus souvent sur les paramètres suivant : degré, diamètre, connectivité et extension. Cependant, à des types précis de matrices de trafic et à des routages particuliers, correspondent probablement des topologies idoines. Le facteur topologique d'un graphe, qui n'est autre que le reflet de l'analyse structurelle du graphe découlant de son utilisation, permet un choix optimal de graphe.

Partant d'une taille minimale, on calcule pas à pas la variation du (ou des) facteur(s) topologique(s) en fonction de l'extension effectuée. On retient alors l'extension qui minimise les variations de facteurs topologiques pour un type de trafic et un routage donné. Il faut remarquer que la minimisation du diamètre moyen, tout au long de l'extension, est souvent suffisante d'après la remarque 2.6: on réduit en effet l'ordre des ensembles $\mathscr{E}(A, B)$ et la "profondeur» des couches.

En contrepartie on diminue le nombre de PCCH possibles entre 2 nœuds, ce qui peut rendre certaines mailles critiques (voir 4.4).

Exemple : Considérons les deux graphes suivants :

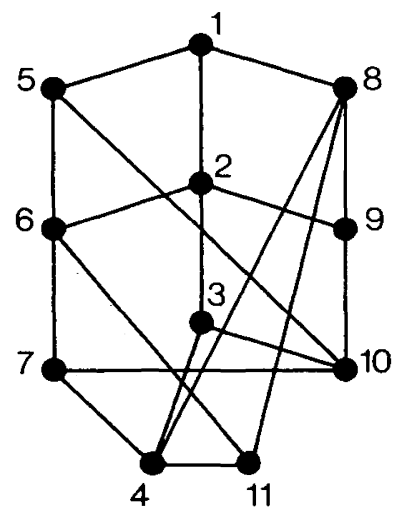

GRAPHE 1

$N=11$

$P=4$

$T=3$

$\bar{P}=3,45$

$\bar{T}=1,71$

$M=19$

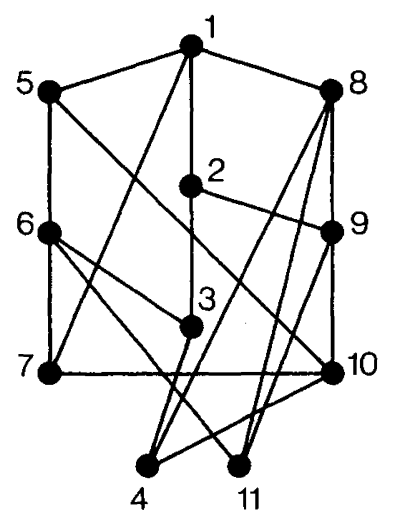

GRAPHE 2

$N=11$

$P=4$

$T=2$

$\overline{\mathrm{P}}=1,65$

$\bar{T}=3,45$

$M=19$ 


\begin{tabular}{|l|l|l|l|l|l|l|l|l|l|}
\hline \multirow{2}{*}{} & & \multicolumn{4}{|c|}{ Trafic équiréparti } & \multicolumn{4}{c|}{ Trafic pessimiste } \\
\cline { 3 - 10 } & & $A_{\mathbf{0}}$ & $D_{0}$ & $B_{\mathbf{0}}$ & $C_{0}$ & $A_{2}$ & $D_{2}$ & $B_{2}$ & $C_{2}$ \\
\hline \multirow{2}{*}{ Graphe 1 $\ldots$} & Maxi. & 0,9 & 0,6 & 0,625 & 0,5 & 3 & 3 & 2,25 & 2 \\
\cline { 3 - 11 } & Moy. & 0,763 & 0,4947 & 0,4947 & 0,32 & 2,63 & 2,1 & 1,87 & 1,58 \\
\hline \multirow{2}{*}{ Graphe 2 $\ldots$} & Maxi. & 0,7 & 0,6 & 0,6 & 0,6 & 2 & 2 & 2 & 2 \\
\cline { 3 - 11 } & Moy. & 0,574 & 0,4789 & 0,4789 & 0,39 & 2 & 1,95 & 1,82 & 1,68 \\
\hline
\end{tabular}

Le graphe 2 est un graphe optimisé pour le diamètre moyen et construit à partir d'un graphe de Petersen [14]. Il ne diffère du graphe 1 que par 4 mailles et conserve le même degré moyen et le même nombre de mailles.

On constate que seuls $C_{0}$ et $C_{2}$ (hypothèses du routage optimiste) sont moins bons. Cela s'explique en effet par le fait qu'un graphe optimisant le diamètre moyen laisse peu de possibilité au routage; il n'existe souvent qu'un seul plus court chemin entre 2 nœuds, et chaque maille est donc utilisée au maximum.

En revanche, le trafic de transit est plus faible ce qui améliore la charge des mailles.

\subsection{Mailles critiques. Nœuds critiques}

Une maille peut être considérée comme critique sous plusieurs aspects. Si l'on suppose connu l'algorithme de routage et la matrice de trafic échangé, quatre définitions sont en effet possibles.

- soit $F$ le facteur topologique du graphe considéré et $F_{\underline{A B}}$ le facteur obtenu lorsque la maille $A B$ est déconnectée. La maille $A B$ est d'autant plus critique que $F_{\underline{A B}} / F$ est grand;

- lorsqu'une maille est nécessairement empruntée par des plus courts chemins, elle est d'autant plus critique que le facteur $F\left(T_{i}, R_{4}\right)$ est élevé.

Ce critère doit cependant être complété :

- une maille autorisant un couplage de cardinal $D_{2}$ entre ses origines et ses extrémités est d'autant plus critique que $D_{2}$ est proche de $Q_{M}$ (borne de Moore).

Enfin :

- une maille est d'autant plus critique que le facteur topologique relatif à cette maille est supérieur à la moyenne.

La modélisation d'un réseau par ses facteurs topologiques permet de choisir un routage ou un graphe support permettant de limiter les mailles critiques.

Un nœud critique est un nœud qui, déconnecté du graphe, entraîne l'apparition de mailles critiques.

vol. $22, \mathrm{n}^{\circ} 2,1988$ 


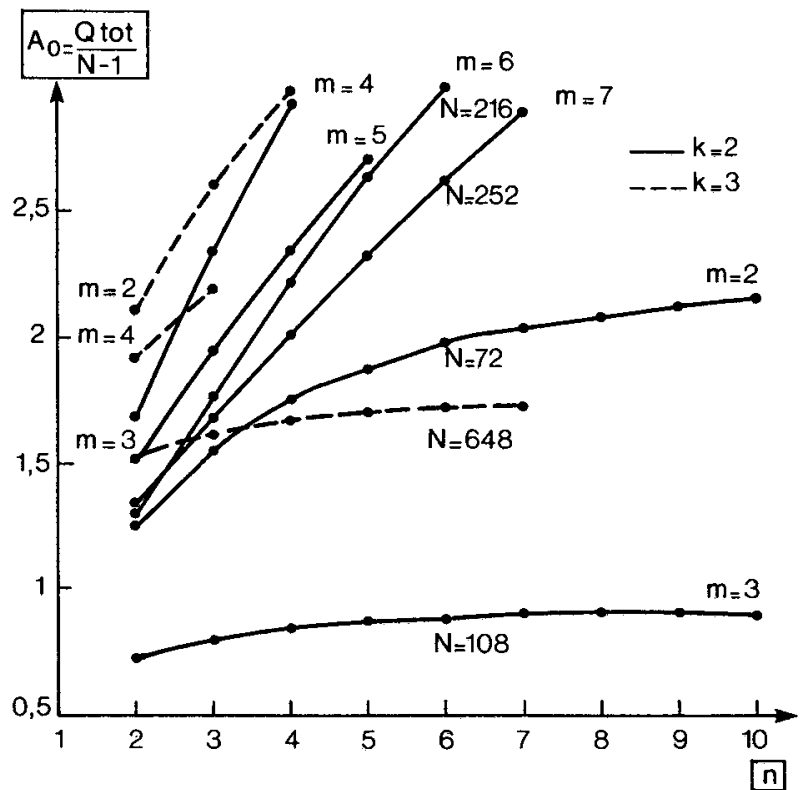

Figure 7

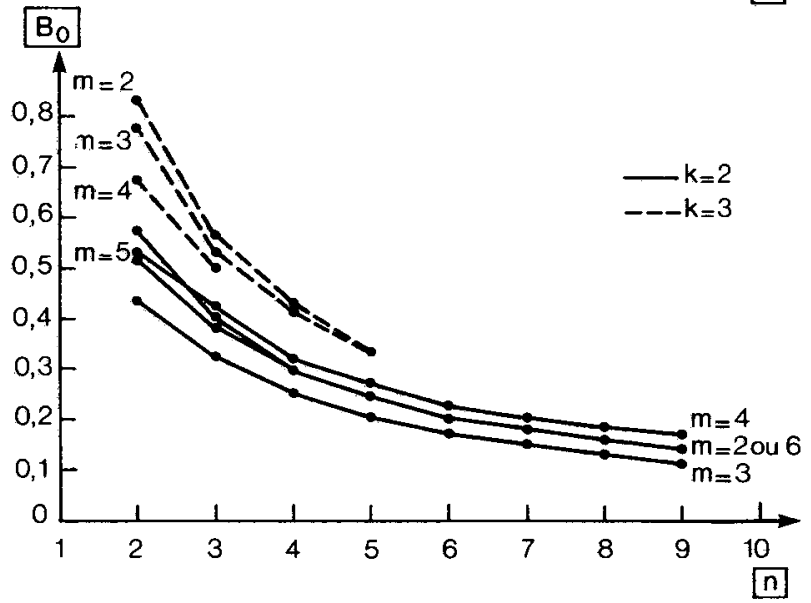

Figure 8

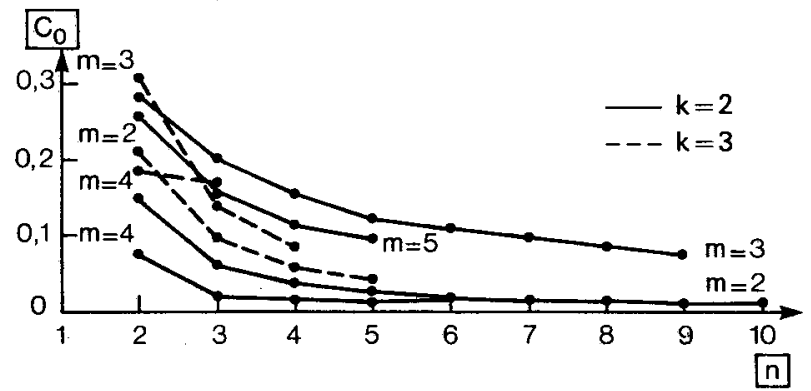

Figure 9

Recherche opérationnelle/Operations Research 


\section{5. Études particulières d'une classe de graphe}

Une application possible de la modélisation d'un graphe par ses facteurs topologiques consiste précisément à étudier l'évolution des quatre facteurs simples $A_{0}, B_{0}, C_{0}$ et $Q$, en fonction du nombre de nœuds et de la structure du graphe.

Un type de graphe se prête particulièrement à cette étude: les graphes $C_{s}[14]$ construits sur l'idée des graphes alphabets et caractérisés par trois facteurs $(k, m, n)$. Le nombre de nœuds $N$ vaut $N=m . n^{k}$.

Pour ces graphes et dans le cas d'un trafic équiréparti, lorsque $n>3$, toutes les arêtes d'un même graphe ont des facteurs topologiques $\left(A_{0}, B_{0}, C_{0}\right)$ identiques si $m \geqq 1$. Le facteur $B_{0}$, au contraire décroît avec $n, k$ et $m$ fixés. Ce facteur est rigoureusement égal à $\bar{T} / \bar{p}$ (voir fig. $7,8,9$ ).

Ces courbes permettent de choisir, dans une même classe de graphe celui qui autorise le nombre maximal de nœuds connectés avec $A_{0}, B_{0}$ et $C_{0}$ minimaux.

\section{CONCLUSION}

Dans le cas de réseaux permettant à des nœuds d'échanger du trafic, la modélisation des charges de mailles inter-nœud est possible grâce aux facteurs topologiques.

Ces facteurs résument globalement ou pour chaque maille, le type de routage adopté, la matrice des trafics échangés et la structure du graphe. Ainsi, connaissant les hypothèses de fonctionnement du réseau, on obtient pour chaque maille et par simple multiplication du débit injecté par tous les nœuds, le débit réel s'écoulant sur les mailles.

Un certain nombre d'applications sont possibles : choix d'un graphe, étude particulière d'une classe de graphe, caractérisation des mailles etc. La modélisation d'un graphe par ses facteurs topologiques nécessite néanmoins des connaissances précises quant au trafic et au routage. Le cas du trafic équiréparti donne une approximation non biaisée de la charge des mailles.

\section{ANNEXE 1}

\section{DEMONSTRATION DU RESULTAT 1}

Soit $(X, Y)$ un $\mathrm{PCCH}$ utilisant $(A, B)$ de longueur $k$. D'après le théorème de Bellman Kalaba [15], $(X, A)$ et $(Y, B)$ sont des PCCH. 
Si $d(X, A)=i$ et $d(Y, B)=j$ avec $k=i+j+1$, alors

$$
X \in \mathscr{D}_{A}(i) \quad \text { et } \quad Y \in \mathscr{D}_{B}(j)
$$

$X$ n'est pas élément de $\mathscr{D}^{*} B(i-1)$ : en effet si $X \in \mathscr{D}_{B}^{*}(i-1)$ alors $X \in \mathscr{D}_{B}(i-1)$ et $d(X, Y)=i-1+j \neq k$.

Donc $X \in \mathscr{D}_{A}^{*}(i)=\mathscr{D}_{A(i)}-\mathscr{D}_{B}^{*}(i-1)$.

De même $Y \in \mathscr{D}_{B}^{*}(j)=\mathscr{D}_{B}(j)-\mathscr{D}_{A}^{*}(j-1)$.

\section{DÉMONSTRATION DU RÉSULTAT 2}

- $\mathscr{D}_{A}^{*}(i) \cap \mathscr{D}_{B}^{*}(j)=\varnothing$ par définition si $i \neq j$;

- soit $X \in \mathscr{D}_{A}^{*}(i) \cap \mathscr{D}_{B}^{*}(j)$

$X \in \mathscr{D}_{A}^{*}(i)$ : il existe $z$ tel que $(X, z)$ admet un PCCH utilisant $(A, B)$

$X \in \mathscr{D}_{B}^{*}(j):$ il existe $z^{\prime}$ tel que $\left(z^{\prime}(X)\right.$ admet un PCCH utilisant $(A, B)$

$$
\begin{aligned}
d(X, z) & =d(X, A)+1+d(B, z) \leqq d(X, B)+d(B, z) \\
d\left(z^{\prime} X\right) & =d\left(z^{\prime} A\right)+1+d(B, X) \leqq d\left(z^{\prime} A\right)+d(A, X)
\end{aligned}
$$

soit

$$
\left\{\begin{array}{l}
i+1 \leqq j \\
j+1 \leqq i
\end{array}\right. \text { ce qui est impossible. }
$$

\section{BIBLIOGRAPHIE}

1. Spécial Issue on Interconnexion Networks, Computer, 12/81.

2. La revue des Télécommunications/ITT, $\mathrm{n}^{\circ} 56,2 / 3$.

3. Duret-Henry, Système de commutation à structure nodale, CIC/ISS, 1981, Montréal, 21B4.

4. Dias-Jump, Packet Switching Interconnection Network, Computer, 12/81, p. 43-53.

5. TURNer, Design of a Broadcast Packet Switching Network, I.E.E.E., 1986 (à paraitre).

6. CoudReuse, Les réseaux temporels asynchrones du transfert de donnée à l'image animée, Echo des Recherches, $\mathrm{n}^{\circ} 112,1983$.

7. Devault et al., Commutation temporelle asynchrone, CIC/ISS, 1981, Montréal, $42 \mathrm{~B} 5$.

8. Servel, Réseaux de transfert en videcommunication commutation de paquets, Echo des Recherches, $\mathrm{n}^{\circ} 115,1984$. 
9. Documents parus dans le cadre de RACE/1024/WP4.

10. KLEINRock, Queuing Systems, vol. 1, John Wiley, 1975.

11. Dieudonne et al., Expérimentation de nouveaux Concepts d'architecture d'autocommutateurs R NIS, Revue Thomson, vol. 16, $\mathrm{n}^{\circ}$ 3, 9/1984.

12. RaIllard, Réseaux nodaux, CIC/ISS, 1981, Montréal, 14A5.

13. BERGE, Graphes et hypergraphes, Dunod, Vol. 604, 1973.

14. Delorme, Large Graphs with Given Degree and Diameter, Part 1, I.E.E.E.-Trans on Comp., vol. C33, n ${ }^{\circ}$ 9/1984.

15. Gondran, Graphes et Algorithmes, Eyrolles, 1979.

16. Bubenix-Turner, Performance of a Broadcast Packet Switching Network, WUCS86-10, 6/1986.

17. Bollobas, External Graph Theory, London Math. Soc., Monographs, ${ }^{\circ} 11$, Academic Press, London, 1978.

18. TURner, Revue Globecom, A Packet Architecture for Integrated Services, Vol. 181983, 211-216.

19. Lalanne, Descriptions générales des caractéristiques d'un réseau RNA, Rapport Alcatel, 30483502 TAA. 